\title{
Retinoblastoma by AJCC v8 Stage
}

National Cancer Institute

\section{Source}

National Cancer Institute. Retinoblastoma by A/CC v8 Stage. NCI Thesaurus. Code C140750.

A term that refers to the staging of retinoblastoma according to the American Joint Committee on Cancer, 8th edition. This staging system does not apply to central nervous system component of "trilateral retinoblastoma" (is staged according to the classification for brain and spinal cord), retinoma (or retinocytoma) (no AJCC staging system), and medulloepithelioma (no AJCC staging system). (from AJCC 8th Ed.) 